Article

\title{
Cost-Effectiveness of the Manchester Approach to Identifying Lynch Syndrome in Women with Endometrial Cancer
}

\author{
Tristan M. Snowsill ${ }^{1, *(\mathbb{C},}$, Neil A. J. Ryan ${ }^{2,3,4}$ (1) and Emma J. Crosbie ${ }^{3,5}$ (1) \\ 1 Health Economics Group, University of Exeter Medical School, Exeter EX1 2LU, UK \\ 2 Division of Evolution and Genomic Medicine, University of Manchester, St Mary's Hospital, \\ Manchester M13 9WL, UK; neilryan@nhs.net \\ 3 Division of Cancer Sciences, Faculty of Biology, Medicine and Health, University of Manchester, \\ St Mary's Hospital, Manchester M13 9WL, UK; emma.crosbie@manchester.ac.uk \\ 4 Academic Centre for Women's Health, University of Bristol, Bristol BS8 2PS, UK \\ 5 Division of Gynaecology, Manchester University NHS Foundation Trust, Manchester Academic Health \\ Science Centre, Manchester M13 9WL, UK \\ * Correspondence: t.m.snowsill@exeter.ac.uk
}

Received: 25 April 2020; Accepted: 22 May 2020; Published: 1 June 2020

check for updates

\begin{abstract}
Lynch syndrome (LS) is a hereditary cancer syndrome responsible for $3 \%$ of all endometrial cancer and 5\% in those aged under 70 years. It is unclear whether universal testing for LS in endometrial cancer patients would be cost-effective. The Manchester approach to identifying LS in endometrial cancer patients uses immunohistochemistry (IHC) to detect mismatch repair (MMR) deficiency, incorporates testing for MLH1 promoter hypermethylation, and incorporates genetic testing for pathogenic MMR variants. We aimed to assess the cost-effectiveness of the Manchester approach on the basis of primary research data from clinical practice in Manchester. The Proportion of Endometrial Tumours Associated with Lynch Syndrome (PETALS) study informed estimates of diagnostic performances for a number of different strategies. A recent microcosting study was adapted and was used to estimate diagnostic costs. A Markov model was used to predict long-term costs and health outcomes (measured in quality-adjusted life years, QALYs) for individuals and their relatives. Bootstrapping and probabilistic sensitivity analysis were used to estimate the uncertainty in cost-effectiveness. The Manchester approach dominated other reflex testing strategies when considering diagnostic costs and Lynch syndrome cases identified. When considering long-term costs and QALYs the Manchester approach was the optimal strategy, costing £5459 per QALY gained (compared to thresholds of $£ 20,000$ to $£ 30,000$ per QALY commonly used in the National Health Service (NHS)). Cost-effectiveness is not an argument for restricting testing to younger patients or those with a strong family history. Universal testing for Lynch syndrome in endometrial cancer patients is expected to be cost-effective in the U.K. (NHS), and the Manchester approach is expected to be the optimal testing strategy.
\end{abstract}

Keywords: Lynch syndrome; endometrial cancer; reflex testing; cost-effectiveness analysis; decision analytic model

\section{Introduction}

Lynch syndrome is an inherited cancer predisposition syndrome caused by pathogenic variants in the DNA mismatch repair (MMR) genes. It has incomplete penetrance, which is also dependent on which MMR gene is affected, but most individuals with Lynch syndrome face much higher risks of colorectal cancer, endometrial cancer, and ovarian cancer than the general population [1]. There are 
generally no signs or symptoms of Lynch syndrome other than developing cancer. An individual is generally regarded as having Lynch syndrome if they have a pathogenic MMR variant (path_MMR) regardless of whether they have been diagnosed with an associated cancer.

As individuals with Lynch syndrome are more likely to develop colorectal and endometrial cancer, the prevalence of Lynch syndrome is higher amongst those diagnosed with these cancers than in the general population. Further, it is possible to test colorectal and endometrial tumours for signs that Lynch syndrome may be implicated, as mismatch repair deficiency (dMMR) is associated with Lynch syndrome and can be detected through molecular testing for microsatellite instability (MSI) or through immunohistochemistry (IHC). Additionally, testing for hypermethylation of the MLH1 promoter region is able to rule out some sporadic cancers that demonstrate dMMR. Reflex (automatic) testing of colorectal and endometrial cancer cases for possible Lynch syndrome, followed by referral for diagnostic genetic testing, is a proven strategy for identifying individuals (and their family members) with Lynch syndrome [2,3].

At present, testing for Lynch syndrome in endometrial cancer patients is not widespread in the U.K. The U.K. health service is dominated by the National Health Service (NHS), which provides healthcare mostly free of charge, paid for through general taxation (i.e., users are generally not required to purchase social or private medical insurance). The different nations of the U.K. (England, Scotland, Wales, and Northern Ireland) have different organisational systems, but in England (which accounts for over $80 \%$ of the U.K. population) funding for healthcare mostly flows from the Department of Health and Social Care, through NHS England, to regional clinical commissioning groups (CCGs) on a capitation basis, and then onto hospitals and other providers on the basis of activity following a national tariff. The National Institute for Health and Care Excellence (NICE) is currently assessing the effectiveness and cost-effectiveness of such testing, but this paper presents an independent economic evaluation not connected to the NICE assessment.

A number of studies have shown that reflex testing of colorectal cancer cases for Lynch syndrome is effective and also cost-effective [4-8]. Demonstrating cost-effectiveness for reflex testing is important to enable testing to be covered/reimbursed. More recent evidence has emerged that reflex testing of endometrial cancer cases for Lynch syndrome may also be cost-effective [9-13], but much of this evidence is limited in its scope, for example, most have not included the option of not testing for Lynch syndrome as a comparator and have only compared testing strategies [9-12]. We recently published a model-based economic evaluation using prevalence and diagnostic accuracy data estimated from published literature [13], but this cannot adequately address that different testing strategies may not only have different performance characteristics, but may also identify different Lynch syndrome cases and face different challenges in a heterogeneous population. For example, MSI testing may both be less specific in older patients (where somatic MLH1 hypermethylation is known to be more common in colorectal cancer [14]) and may be less sensitive to identify path_MSH6 and path_PMS2 cases (i.e., pathogenic variants in MSH6 and PMS2) [15].

The Proportion of Endometrial Tumours Associated with Lynch Syndrome (PETALS) study [16] was conducted in a large U.K. gynaecological cancer centre to evaluate the effectiveness of reflex testing in endometrial cancer cases and to estimate the prevalence of Lynch syndrome in this population. A total 500 endometrial cancer cases were tested with IHC and MSI (300 recruited prospectively, 200 identified retrospectively having been consented for research), with MLH1 methylation testing and germline next generation sequencing (NGS) being conducted where indicated. Sixteen women were diagnosed with Lynch syndrome.

For an economic evaluation of reflex testing for Lynch syndrome in endometrial cancer patients to be most valuable for decision makers, it should use reliable, consistent, and unbiased evidence for key diagnostic performance parameters, and it should account for the differing values of identifying Lynch syndrome cases according to their age and genotype. 


\section{Methods}

We performed an economic evaluation on the basis of patient-level data from the PETALS study [16]. For each participant in PETALS, we simulated their diagnostic outcomes (and associated costs) according to different diagnostic strategies. We used a Markov model to extrapolate the expected lifetime costs and health outcomes for each participant on the basis of their diagnostic outcome. These were combined, along with estimated costs and benefits of cascade testing for Lynch syndrome in relatives of confirmed cases, to estimate the expected lifetime costs and quality-adjusted life years (QALYs) for each diagnostic strategy, as well as to perform a cost-effectiveness analysis (see Table 1) to determine the optimal strategy.

Table 1. Economic evaluation characteristics.

\begin{tabular}{|c|c|}
\hline Decision Problem & $\begin{array}{l}\text { What is the Relative Cost-Effectiveness of Strategies to Identify Lynch } \\
\text { Syndrome in Women with Endometrial Cancer }\end{array}$ \\
\hline \multirow{7}{*}{ Interventions and comparators } & Strategy 0: No testing \\
\hline & Strategy 1: MSI triage followed by NGS \\
\hline & Strategy 2: MSI and MLH1 methylation testing triage followed by NGS \\
\hline & Strategy 3: IHC and MLH1 methylation testing triage followed by NGS (the \\
\hline & Manchester approach) \\
\hline & Strategy 4: Direct NGS \\
\hline & $\begin{array}{l}\text { Cost-effectiveness analysis: Diagnostic costs and Lynch syndrome cases identified } \\
\text { (no cost-effectiveness threshold identified) }\end{array}$ \\
\hline $\begin{array}{l}\text { Type of economic evaluation, costs, } \\
\text { and health outcomes }\end{array}$ & $\begin{array}{l}\text { Cost-utility analysis: Lifetime costs and QALYs for women with endometrial } \\
\text { cancer and their relatives (cost-effectiveness threshold } £ 20,000 \text { per QALY) }\end{array}$ \\
\hline Model type & Decision tree and Markov model implemented in R \\
\hline Key data source & PETALS study (diagnostic accuracy study conducted in Manchester) \\
\hline Perspective & NHS and PSS, costs in pounds sterling ( $£$; GBP) in $2016 / 17$ prices \\
\hline Time horizon & Lifetime \\
\hline Discounting & $3.5 \%$ for costs and QALYs \\
\hline Analysis of uncertainty & $\begin{array}{l}\text { Non-parametric bootstrap resampling of participants in a clinical study and } \\
\text { parametric sampling of model parameters (probabilistic sensitivity analysis) }\end{array}$ \\
\hline
\end{tabular}

MSI: microsatellite instability; IHC: immunohistochemistry; NGS: next generation sequencing; QALY: qualityadjusted life years; PETALS: The Proportion of Endometrial Tumours Associated with Lynch Syndrome; NHS: National Health Service; PSS: personal social services; GBP: (Great British) Pounds sterling (£).

\subsection{Selection of Interventions and Comparators}

\subsubsection{The Manchester Approach}

The Manchester approach uses a four-MMR protein immunohistochemistry panel to triage all endometrial cancer cases. Where no assays indicate loss of expression, the case is discharged from further testing for Lynch syndrome. If the tumour shows loss of MLH1 and/or PMS2 (but MSH2 and MSH6 are expressed), MLH1 methylation testing is conducted on DNA from tumour cells and from normal adjacent tissue. Cases showing hypermethylation in tumour cells are discharged from further testing for Lynch syndrome. If the tumour shows loss of MSH2 and/or MSH6, or if the tumour shows loss of MLH1 and/or PMS2 expression unexplained by MLH1 methylation, a NGS panel is used to identify constitutional pathogenic variants in the MMR genes (and EPCAM).

Informed consent is obtained in the gynaecology clinic prior to NGS testing, but only patients in whom a pathogenic MMR variant is found are referred for genetic counselling.

\subsubsection{Comparator Strategies}

The principal comparators to the Manchester approach (which is labelled strategy 3) that were included in this evaluation are (see Figure 1a):

- No testing for Lynch syndrome in endometrial cancer cases (strategy 0);

- Triage by microsatellite instability testing, followed by NGS (strategy 1); 
- Triage by microsatellite instability testing, then MLH1 methylation testing, followed by NGS (strategy 2);

- $\quad$ Direct NGS (strategy 4).

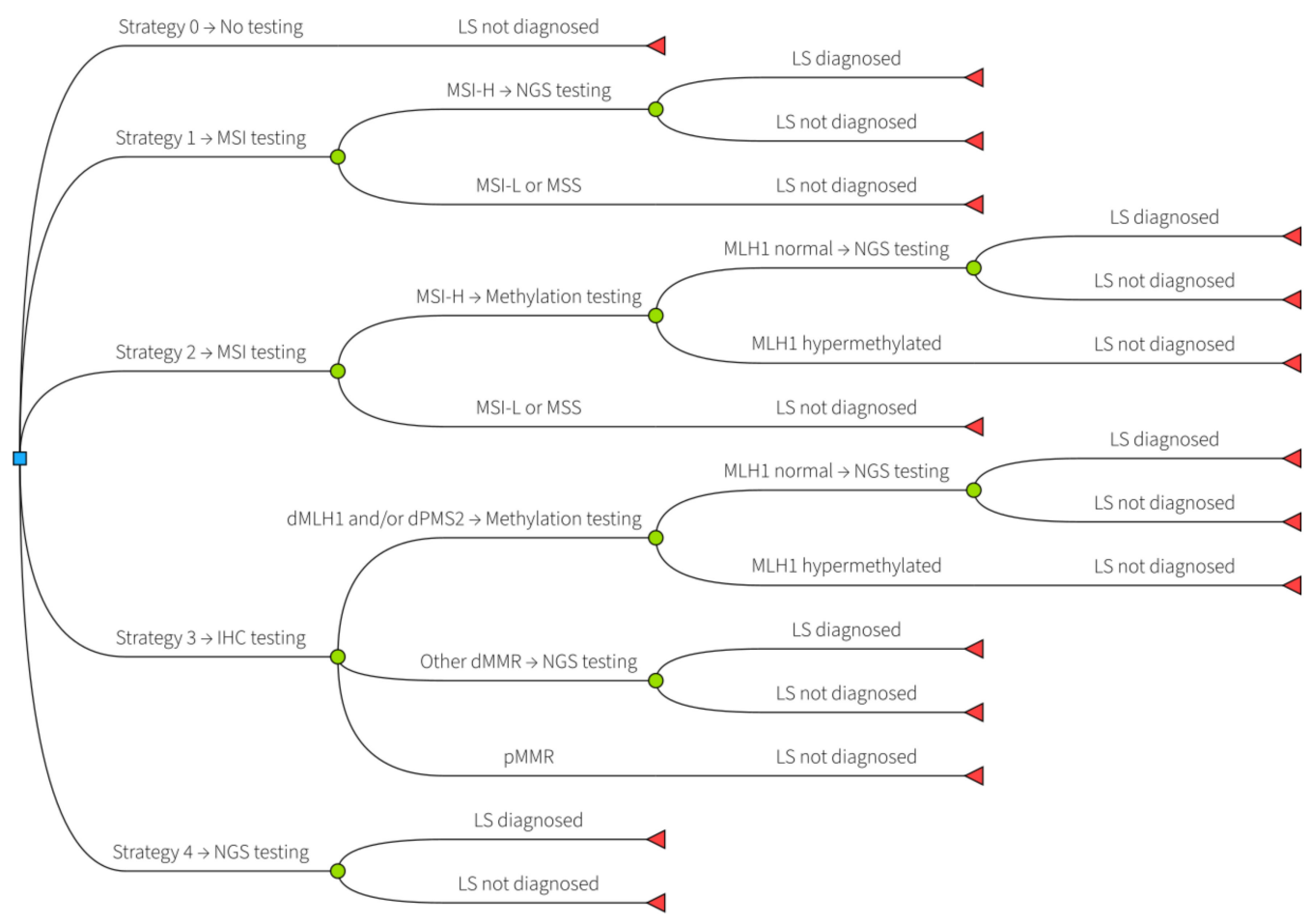

(a)

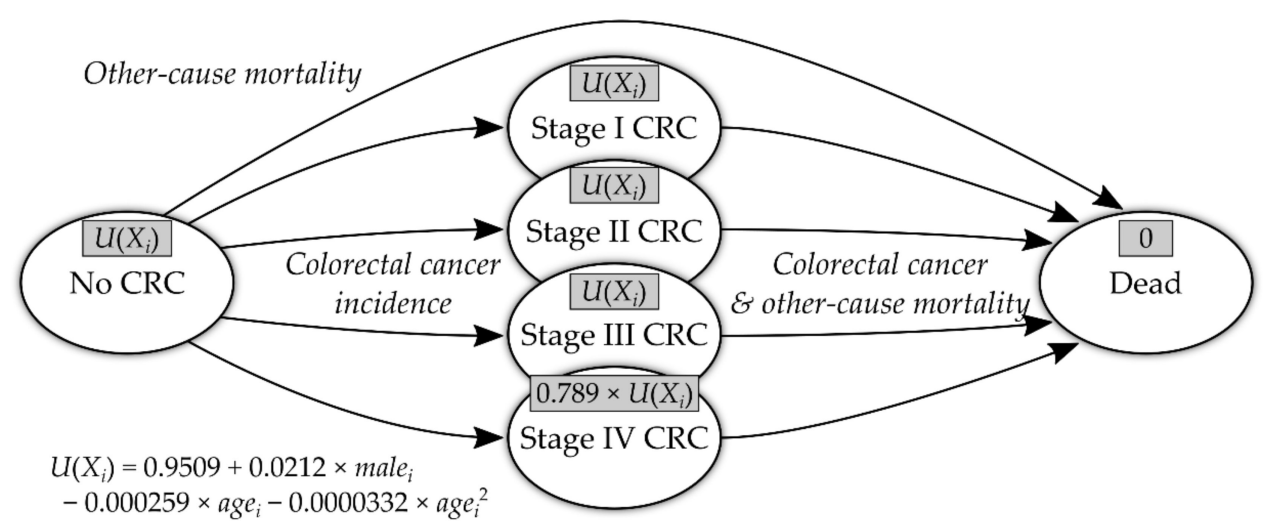

(b)

Figure 1. Model diagrams: (a) interventions and comparators in the economic evaluation; LS: Lynch syndrome; MSI: microsatellite instability; MSI-H: microsatellite instability—high; MSI-L; microsatellite instability-low; NGS: next generation sequencing; MSS: microsatellite stable; IHC: immunohistochemistry; dMLH1: deficient MLH1 expression; dPMS2: deficient PMS2 expression; dMMR: mismatch repair deficiency; pMMR: proficient mismatch repair. (b) Markov model structure (self-links not shown for clarity); grey boxes in each health state give the utility value (QALY (quality-adjusted life years) weight) for that state; $\mathrm{U}\left(\mathrm{X}_{\mathrm{i}}\right)$ : utility value for individual I with characteristics $X_{i}$; CRC: colorectal cancer.

Microsatellite instability testing typically includes a panel of five markers, and instability at two or more of these is classed as microsatellite instability—high (MSI-H). Instability at a single marker 
is referred to as microsatellite instability-low (MSI-L). Instability in zero markers is referred to as microsatellite stable (MSS). MSI is frequently caused by MLH1 promoter hypermethylation, but the MSI markers have no diagnostic utility for recognising this, and thus either all MSI cases must undergo methylation testing or none.

In addition to these comparators, we explored certain variations in scenario analyses:

- Using a two-MMR protein IHC panel (including only MSH6 and PMS2 antibodies);

- Treating MSI-L as indicative for further Lynch syndrome testing;

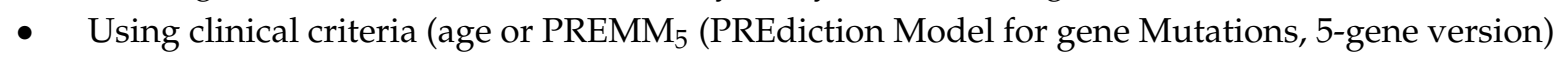
score [17]) to select patients at higher risk of having Lynch syndrome.

\subsection{Economic Evaluation Approach}

\subsubsection{Diagnostic Testing}

For each participant in the PETALS study, we simulated their diagnostic pathway according to each diagnostic strategy. For example, if the tumour of a patient in PETALS had absent MSH6 but was MSS, they would be simulated as discharged from further testing in strategies 1 and 2, even though in PETALS they would have received further testing. Where MLH1 methylation testing was not ordered in PETALS (on the basis of IHC results), it was assumed the test would not have shown hypermethylation. Patients not undergoing NGS in PETALS were assumed not to have Lynch syndrome.

When an endometrial cancer patient (proband) is diagnosed with Lynch syndrome, cascade testing of their relatives is simulated. For each proband, we modelled on average six relatives having an appointment with a general practitioner in relation to Lynch syndrome, of whom 4.7 attended genetic counselling, 3.3 underwent predictive genetic testing, and 1.5 were diagnosed with Lynch syndrome [13]. The relatives were modelled irrespective of the true Lynch syndrome status and diagnostic outcome for the proband, so that there was a consistent population across interventions.

\subsubsection{Extrapolation}

A Markov model was used to extrapolate future costs and health outcomes (measured in QALYs). The Markov model was adapted from the model used by Snowsill et al. [13]. It was ported from Excel to R [18] and built using the heemod package [19].

The Markov model included colorectal cancer as a downstream consequence of having Lynch syndrome (diagnosed or undiagnosed) and colonoscopy as a risk-reducing intervention for those with diagnosed Lynch syndrome. The incidence of colorectal cancer is modelled on the basis of which (if any) MMR gene contains a pathogenic variant and the age of the individual [13]. For relatives, the sex also influences the incidence of colorectal cancer [13]. Colonoscopy has been assumed to directly reduce the incidence of colorectal cancer $[8,20]$ and to make detection of colorectal cancer in earlier stages more likely $[8,21]$. Colorectal cancer survival was modelled in terms of stage, with stage IV having the worst prognosis and stage I the best. A model diagram is shown in Figure $1 \mathrm{~b}$.

The Markov model does not incorporate other Lynch syndrome-associated cancers, such as future gynaecological cancer, cancers of the non-colorectal digestive tract, and cancers of the biliary and urinary tracts [1]. It also does not include other preventative measures such as gynaecological surveillance and aspirin chemoprevention, as these are not routinely used in clinical practice.

\subsection{Methods for Estimating Costs}

Costs are presented in 2016/2017 pounds Sterling ( $£$; GBP), inflated using the Hospital and Community Health Services Pay and Prices Index [22] to 2015/2016 and then by 1.1\% to 2016/2017. 


\subsubsection{Diagnostic Costs}

The microcosting conducted by Ryan et al. [23] provided estimates for the majority of diagnostic costs, however, we adjusted the unit costs to add on costs for staff time (as the original microcosting included only staff salaries). This involved scaling labour costs by a factor of 2.08, and represented additional costs of employers' pension contributions, employers' National Insurance Contributions, and overheads (management, administrative and estates staff, non-staff overheads), but did not include capital overheads. This was estimated by taking the average ratio of total cost (excluding capital overheads) to salary for hospital-based scientific and professional staff in bands 4 to 7 [22].

We assumed that IHC with a two-MMR antibody panel would cost half the cost of the standard test. This likely represents a best case scenario as there are likely to be some economies of scale. It was assumed that filtering cases on the basis of age would not incur any cost. The cost of calculating, recording, and communicating the $\mathrm{PREMM}_{5}$ score for a patient was assumed to be $£ 3.58$ (on the basis of requiring $5 \mathrm{~min}$ of gynaecology registrar time) [22].

Costs for genetic counselling were estimated on the basis of Slade et al. [24], with post-test counselling costing $£ 133$ (for both probands and relatives) and pre-test genetic counselling costing $£ 172$ for relatives. The Manchester approach follows the mainstreaming cancer genetics paradigm of not offering pre-test genetic counselling for probands [25]. As a scenario analysis, we assumed counselling costs of $£ 428$ for the first visit (post-test counselling for probands, pre-test counselling for relatives) and $£ 403$ for a follow-up visit (post-test counselling for relatives) [26].

A summary of diagnostic costs is given in Table 2 .

Table 2. Diagnostic testing unit costs.

\begin{tabular}{ccc}
\hline Item & Unit Cost (£, GBP) & Source \\
\hline Calculate PREMM 5 score & 3.58 & PSSRU 2017 [22] \\
MMR IHC (4 protein panel) & $30.36^{1}$ & Ryan et al. 2019 [23] \\
MMR IHC (2 protein panel) & 15.18 & Assumed half cost of 4 protein panel \\
MSI testing & $36.63^{1}$ & Ryan et al. 2019 [23] \\
MLH1 methylation testing (strategy 2) & $22.84^{1}$ & Ryan et al. 2019 [23] \\
MLH1 methylation testing (strategy 3) & $32.65^{1}$ & Ryan et al. 2019 [23] \\
Obtain consent for NGS & $13.64^{1}$ & Ryan et al. 2019 [23] \\
NGS & $236.35^{1}$ & Ryan et al. 2019 [23] \\
Post-test genetic counselling (probands) & 133.15 & Slade et al. 2016 [24] \\
Pre-test genetic counselling (relatives) & 171.73 & Slade et al. 2016 [24] \\
Predictive genetic testing (relatives) & 166.32 & Slade et al. 2016 [24] \\
Post-test genetic counselling (relatives) & 133.15 & Slade et al. 2016 [24] \\
\hline
\end{tabular}

Key: IHC, immunohistochemistry; MMR, mismatch repair; MSI, microsatellite instability; NGS, next generation sequencing; PREMM $_{5}$ : PREdiction Model for gene Mutations, 5 -gene version. Notes: ${ }^{1}$ Labour costs scaled by 2.08 to include additional costs other than salary.

\subsubsection{Long-Term Costs}

The Markov model used to extrapolate long-term costs and outcomes includes costs for colonoscopic surveillance and costs associated with colorectal cancer. Colonoscopies were assumed to cost $£ 583$ [27], whereas the cost of colorectal cancer was dependent on the age of the patient and stage of cancer at time of diagnosis, ranging from $£ 807$ (aged 80+, stage IV) to $£ 14,490$ (aged 40-49, stage III) [28].

\subsection{Methods for Estimating Health Benefits}

\subsubsection{Lynch Syndrome Cases Identified}

For strategy 0 (no testing), we assumed no Lynch syndrome cases are identified. For strategy 1 (MSI triage), we assumed a true Lynch syndrome case would have been correctly identified if the tumour demonstrated MSI-H. For strategy 2 (MSI and MLH1 methylation triage), we assumed a true 
Lynch syndrome case would have been correctly identified if the tumour demonstrated MSI-H and if methylation testing showed no hypermethylation or was not conducted in the PETALS study (as this would only be the case if IHC showed normal MLH1 expression). For strategy 3 (IHC and MLH1 methylation triage; the Manchester approach) we assumed a true Lynch syndrome case would have been correctly identified if the tumour demonstrated complete (i.e., not patchy) absence of $1+$ MMR proteins, and that MLH1 methylation testing showed no hypermethylation or was not indicated. For strategy 4 (direct NGS), we assumed that all true Lynch syndrome cases would be identified.

\subsubsection{Long-Term QALYs}

The Markov model used to extrapolate long-term costs and outcomes includes a baseline profile for health-related quality of life that declines with age [29], as well as a loss of quality of life for those with stage IV colorectal cancer (utility is scaled by 0.789 , on the basis of a systematic review and meta-regression [30]).

\subsection{Methods for Handling Uncertainty and Heterogeneity}

Our analyses naturally address heterogeneity in the patient population by projecting future costs and outcomes for each participant in the PETALS trial individually on the basis of their age and MMR genotype (sporadic/wildtype, path_MLH1, path_MSH2, path_MSH6, path_PMS2). We additionally modelled a heterogeneous group of relatives by dividing a cohort of 310 relatives from the Wales regional genetics service (personal communication, Dr Ian Frayling, Cardiff University, Cardiff, Wales, U.K., 2012) into age quintiles, calculating the mean age for each of these $(24.5,35.7,45.0,53.2$, and 66.8 years of age, respectively) and simulating outcomes for men and women, starting at each of these ages with each of the MMR genotypes (50 unique combinations).

Uncertainty is principally handled through a combined non-parametric bootstrap and probabilistic sensitivity analysis approach. We performed 200 iterations, and in each of these iterations, the PETALS population was resampled with replacement and the model was re-run for the resampled population using values for each parameter sampled from probability distributions.

We explored a number of important scenario analyses to reflect alternative strategies for service provision or key parameter assumptions. We explored whether it is cost-effective to apply clinical criteria prior to testing, either restricting testing to those diagnosed with endometrial cancer under the age of 70 or to those with a calculated PREMM score $\geq 2.5 \%$. We also explored the cost-effectiveness of testing under a "worst case" scenario, where the effectiveness of colonoscopic surveillance was estimated from an Italian study [31] that showed the worst performance for surveillance in a systematic review [32], and it was interpreted in the least favourable light to produce a hazard ratio for colorectal cancer incidence of 0.929 (compared to a base case assumption of $0.387[8,20]$ ).

Our deterministic analyses utilised the full PETALS population and central estimates for parameter values (and so incorporated heterogeneity but not parameter uncertainty), whereas our probabilistic analyses incorporated heterogeneity and parameter uncertainty, as described above.

\subsection{Further Details of Economic Evaluation}

Table A3 gives further details of the inputs for the economic evaluation.

\section{Results}

\subsection{Lynch Syndrome Cases Identified}

As reported elsewhere [16], 16 Lynch Syndrome cases were identified amongst the 500 endometrial cancer patients. These patients were younger than those with sporadic endometrial cancer-the mean age for Lynch syndrome cases was 54 years versus 63.5 years for sporadic cases; 15 of 16 Lynch syndrome cases were aged under 70 years versus 314 of 484 sporadic cases. Path_MSH6 variants accounted for eight cases, whereas there were four path_MSH2 variants and two each of path_MLH1 
and path_PMS2. Detailed family history was available for 299 patients and allowed calculation of PREMM $_{5}$ score [17]; 11 of 13 Lynch syndrome cases with detailed family history had PREMM $2 \geq 2.5 \%$, as did 153 of $286(53 \%)$ sporadic cases.

\subsection{Cost per Lynch Syndrome Case Identified}

In the PETALS study there were 16 cases of Lynch syndrome identified. Strategy 0 (no testing) would not be expected to identify any of these, but would incur no cost. Strategies 1 and 2 (MSI-based strategies) would be expected to identify nine of these, whereas strategy 3 (the Manchester approach) and strategy 4 (direct NGS) would be expected to identify all of them. The Manchester approach was the least expensive testing strategy and identified the most cases (tied with direct NGS, which was the most expensive strategy). The MSI-based strategies were dominated by (more expensive and less effective than) the Manchester approach and therefore not predicted to be cost-effective. The Manchester approach was estimated to incur costs of $£ 1700$ per case of Lynch syndrome identified (i.e., an ICER of $£ 1700$ per Lynch syndrome (LS) case identified). Table 3 shows the costs and cases identified for each strategy.

Table 3. Deterministic base case cost-effectiveness analysis.

\begin{tabular}{|c|c|c|c|}
\hline Strategy & Costs $(£)$ & Effectiveness Outcome & ICER \\
\hline Short-term & $\begin{array}{c}\text { Diagnostic pathway } \\
\text { costs } 1\end{array}$ & $\begin{array}{c}\text { Lynch syndrome cases } \\
\text { identified }^{1}\end{array}$ & $\begin{array}{c}\text { Additional diagnostic } \\
\text { pathway cost per Lynch } \\
\text { syndrome case identified }\end{array}$ \\
\hline $\begin{array}{l}\text { Strategy } 0 \\
\text { No testing }\end{array}$ & 0 & 0 & - \\
\hline $\begin{array}{l}\text { Strategy } 1 \\
\text { MSI }\end{array}$ & 41,512 & 9 & Dominated \\
\hline $\begin{array}{c}\text { Strategy } 2 \\
\text { MSI and } M L H 1 \text { methylation }\end{array}$ & 27,523 & 9 & Dominated \\
\hline $\begin{array}{c}\text { Strategy } 3 \\
\text { IHC and } M L H 1 \text { methylation }\end{array}$ & 27,183 & 16 & 1699 \\
\hline $\begin{array}{c}\text { Strategy } 4 \\
\text { Direct NGS }\end{array}$ & 127,125 & 16 & Dominated \\
\hline Lifetime & $\begin{array}{c}\text { Lifetime costs } \\
\text { (proband/proband and } \\
\text { relatives) }\end{array}$ & $\begin{array}{c}\text { Lifetime QALYs } \\
\text { (proband/proband and } \\
\text { relatives) }\end{array}$ & $\begin{array}{l}\text { Additional cost per QALY } \\
\text { gained (proband } \\
\text { only/proband and relatives) }\end{array}$ \\
\hline Strategy 0 & 120 & 7.64 & - \\
\hline No testing & 642 & 104.61 & - \\
\hline Strategy 1 & 223 & 7.65 & Dominated \\
\hline MSI & 842 & 104.65 & Dominated \\
\hline Strategy 2 & 195 & 7.65 & Extendedly dominated \\
\hline MSI and MLH1 methylation & 815 & 104.65 & 3738 \\
\hline Strategy 3 & 220 & 7.66 & 5003 \\
\hline IHC and MLH1 methylation & 928 & 104.67 & 5459 \\
\hline Strategy 4 & 419 & 7.66 & Dominated \\
\hline Direct NGS & 1128 & 104.67 & Dominated \\
\hline
\end{tabular}

Key: ICER, incremental cost-effectiveness ratio; IHC, immunohistochemistry; MSI, microsatellite instability; NGS, next generation sequencing; QALY, quality-adjusted life year. Notes: ${ }^{1}$ Calculated on the basis of PETALS population (500 endometrial cancer patients). Strategies are arranged in ascending order of effectiveness. ICERs were calculated versus the next most effective strategy on the cost-effectiveness frontier.

In the combined bootstrap and probabilistic sensitivity analysis, we found that the $95 \%$ credible interval for the ICER of the Manchester approach versus no testing was $£ 1117$ to $£ 3275$ per LS case identified, and that the Manchester approach dominated the other testing strategies with a probability of 0.475 .

When limiting to endometrial cancer cases aged $<70$ years, the Manchester approach identified 15 Lynch syndrome cases (100\% of those aged < 70 years). Strategy 2 (MSI and MLH1 methylation testing triage) was the cheapest strategy, but only identified nine (60\%) Lynch syndrome cases, and was extendedly dominated by (more expensive and less effective than a combination of) the Manchester 
approach and strategy 0 (no testing). The Manchester approach costed $£ 1300$ per Lynch syndrome case identified if testing only those aged $<70$ years. When comparing universal reflex testing with the Manchester approach to testing only those aged $<70$ years, universal testing costed $£ 7900$ per additional Lynch syndrome case detected.

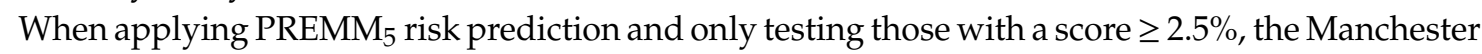
approach identified 11 (100\%) Lynch syndrome cases. Strategy 2 (MSI and MLH1 methylation testing triage) was the cheapest strategy but only identified eight (73\%) Lynch syndrome cases, and was extendedly dominated by the Manchester approach and no testing. The Manchester approach costed $£ 1100$ per Lynch syndrome case identified when PREMM $_{5}$ risk scoring was incorporated. When comparing universal reflex testing with the Manchester approach to testing with PREMM ${ }_{5}$ risk scoring, universal testing costed $£ 2100$ per additional Lynch syndrome case detected.

We found that IHC with a two-antibody (MSH6 and PMS2) panel would detect all 16 (100\%) Lynch syndrome cases in the PETALS study, and on the basis of the assumption that it would halve the cost of IHC testing, it would also result in cost saving, costing $£ 1200$ per Lynch syndrome case identified.

Including MSI-L as a "test positive" in strategy 2 led to the identification of one additional Lynch syndrome case and the requirement for further unnecessary testing in 20 patients, resulting in an additional cost of $£ 5600$ per Lynch syndrome case identified versus only treating MSI-H as a "test positive". The Manchester approach still dominated MSI-based testing strategies.

\subsection{Impact of Surveillance on Life Expectancy, Colorectal Cancer Outcomes, and QALYS}

The Markov model predicts that identifying Lynch syndrome and putting surveillance in place improves life expectancy, but the gain is dependent on which MMR gene is affected and the age of the individual. For example, a 34-year-old proband with path_MLH1 would be expected to gain 4.3 years in life expectancy, whereas a 69-year-old proband with path_PMS2 would only be expected to gain 0.4 years in life expectancy. The average life expectancy gain would be expected to be 1.8 years amongst the probands with Lynch syndrome who are diagnosed and receive surveillance. The average gain in life expectancy from surveillance for female relatives with Lynch syndrome was found to be 4.0 years, whereas it was found to be 3.5 years for male relatives.

The Markov model predicted that for the 16 women diagnosed with Lynch syndrome in PETALS, surveillance would reduce the expected lifetime number of colorectal cancers from 6.3 to 3.2. This included an increase in the number of stage I colorectal cancers from 1.2 to 2.2 , and a reduction in the number of colorectal cancers diagnosed in later stages (stage II: 3.1 to 0.4 ; stage III: 1.3 to 0.4; stage IV: 0.7 to 0.3 ).

Surveillance was also predicted to improve lifetime QALYs (note that lifetime QALYs are discounted at $3.5 \%$ per year, unlike life expectancy). Surveillance was expected to lead to a gain of 0.6 QALYs on average amongst probands with Lynch syndrome, but this ranged from 0.05 to 1.38 QALYs, depending on age and genotype. The average gain in QALYs from surveillance for relatives with Lynch syndrome was found to be 1.1 QALYs for women and 1.0 QALYs for men, with younger relatives and those with path_MLH1 and path_MSH2 variants benefiting most.

\subsection{Cost per $Q A L Y$}

When diagnostic outcomes were extrapolated into lifetime costs and health outcomes (measured in QALYs) and combined with diagnostic costs, we found that strategies 1 and 4 were not cost-effective. When we fixed model parameters at their central estimates, we predicted that the Manchester approach would be cost-effective (see Table 3). When we considered only the endometrial cancer patients themselves, the Manchester approach incurred on average $£ 100$ additional cost and generated an average 0.020 QALYs, giving an ICER of $£ 5000$ per QALY gained. This gain in QALYs would be inconsequential (it corresponds to around 1 week in perfect health), but it was concentrated in the minority of patients who had Lynch syndrome, who gained, on average, 0.624 QALYs from the Manchester approach. When we also included cascade testing of relatives, the Manchester approach 
incurred, on average,e $£ 286$ additional cost per endometrial cancer patient and had an ICER of $£ 5500$ per QALY (compared to strategy 2, which had an ICER of $£ 3800$ per QALY).

In the combined bootstrap and probabilistic sensitivity analysis, we found that the $95 \%$ credible interval for the ICER of the Manchester approach versus no testing was $£ 700$ to $£ 17,000$ per QALY gained (see Figure 2).

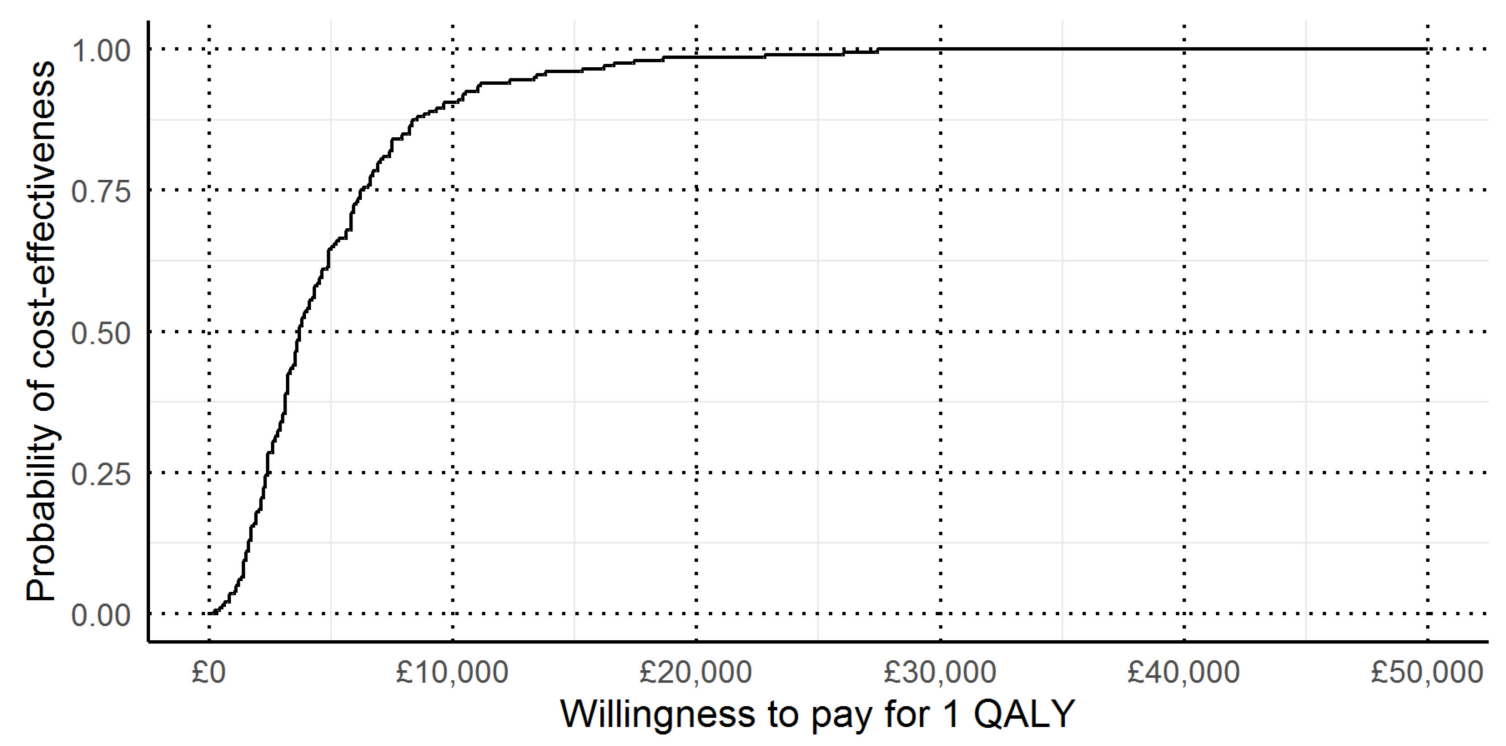

Figure 2. Cost-effectiveness acceptability curve for the Manchester approach versus no testing. This figure shows, on the basis of how much a decision maker is willing to pay for 1 QALY, what the probability is that the Manchester approach is cost-effective compared to no testing.

When testing was restricted to those aged $<70$ years, the Manchester approach and strategy 2 (MSI and MLH1 testing triage) could be cost-effective, depending on the cost-effectiveness threshold. The Manchester approach produced a greater health benefit at a cost of $£ 5500$ per QALY, and was therefore cost-effective at a threshold of $£ 20,000$ per QALY. Comparing universal testing to only testing those aged $<70$ years, universal testing was cost-effective, costing an additional $£ 12,900$ per QALY gained.

When PREMM 5 risk scoring was included (with a threshold of 2.5\%), we found that the Manchester approach was cost-effective, producing the greatest health benefit at an additional cost of $£ 6000$ per QALY gained relative to strategy 2 (the next best option). Universal testing was found to be cost-effective compared to PREMM $_{5}$ testing, costing an additional $£ 11,700$ per QALY gained.

When the effectiveness of colonoscopy was estimated from Arrigoni et al. 2005 [31], the cost-effectiveness of testing worsened, as expected. Strategy 2 was found to be cost-effective at a threshold of $£ 20,000$ per QALY, whereas the Manchester approach was cost-effective with a threshold of $£ 30,000$ per QALY. The Lynch syndrome cases identified by the Manchester approach but not strategy 2 were mainly path_MSH6 cases (5/7) who had a lower risk of colorectal cancer than path_MLH1 and path_MSH2 cases [33,34], and therefore they and their relatives already benefit less in absolute terms from surveillance.

Using IHC with a two-antibody (MSH6 and PMS2) panel would (assuming that no Lynch syndrome cases were missed) save approximately $£ 15$ per patient compared to using the full panel with no change in QALYs.

Including MSI-L as a "test positive" would cost $£ 9500$ per QALY gained compared to only considering MSI-H a "test positive", but would be extendedly dominated by the Manchester approach, and thus would not be cost-effective.

When the cost of genetic counselling was estimated from NHS reference costs for consultant-led clinical genetics outpatient appointments, the Manchester approach remained cost-effective, costing $£ 6700$ 
per QALY gained. The total average lifetime costs per participant (including any costs for relatives) were increased by $£ 60$ over the base case where genetic counselling costs were taken from a costing study for $B R C A$ mutations.

\section{Discussion}

Our study suggests that the Manchester approach is cost-effective compared to no testing and compared to other reflex testing strategies, costing around $£ 5500$ per QALY gained, and further that universal testing (without restricting by age or predicting risk of Lynch syndrome using PREMM P $_{5}$ is cost-effective. We found that restricting IHC testing to a two-antibody (MSH6 and PMS2) panel would improve cost-effectiveness, but this finding was based on no Lynch syndrome cases failing to display deficient expression for one of these proteins within PETALS, whereas other studies have shown that a two-antibody panel is inferior to a four-antibody panel [35]. We found that including MSI-L as a "test positive" result was not sufficient to make MSI-based testing cost-effective compared to the Manchester approach.

The only current published study of reflex testing for Lynch syndrome in women with endometrial cancer to include no testing as a comparator found that IHC and MLH1 methylation triage was cost-effective, but costed approximately $£ 14,000$ per QALY gained for a 60 -year-old endometrial cancer patient, and was not cost-effective for patients aged 65 years and older [13]. The current study based its extrapolation of long-term costs and QALYs on that study, and thus differences in cost-effectiveness were driven by alternative assumptions regarding diagnostic testing, key amongst which are (i) lower costs of IHC (and MSI and MLH1 methylation testing) on the basis of a microcosting study [23] rather than estimates from pathologists and laboratory price lists, and (ii) not including pre-test genetic counselling costs for probands.

This study has a number of strengths. As it was based on a single, large, high-quality trial, it captured all of the associations between patient characteristics and tumour test results, such as the relationship between age and MLH1 hypermethylation, and the possibility of differential test performance for pathogenic variants in different MMR genes. This compares favourably to existing modelling studies that have had to make assumptions about the performance across heterogeneous groups [11-13]. The PETALS study itself has strengths, such as including a U.K. mixed metropolitan population and being conducted in a non-insurance based system, meaning that there were minimal barriers to participants consenting to germline testing [36]. The costs associated with diagnostic testing were measured contemporaneously [23] in the same hospital, which contributed to high internal validity.

The study also has some limitations that should be considered. PETALS identified 16 participants with Lynch syndrome, but there were no cases detectable by MSI but not IHC. Such cases are hypothetically possible and their absence in PETALS means that this possibility was not explored through bootstrapping. Importantly, NGS was not conducted on all PETALS participants (although it was conducted even if tumour tests did not demonstrate MMR deficiency in young patients and those with suggestive family histories), and it was possible that a direct NGS strategy may have identified additional pathogenic MMR variants. Further, as PETALS was a research study, participants were consented for NGS upon entering the study, rather than being consented only after tumour triage testing; it was possible that consenting for NGS at a different time-point could have resulted in lower uptake.

A significant proportion of the value of identifying Lynch syndrome was to implement colonoscopic surveillance to reduce the incidence and mortality due to colorectal cancer, however, the effectiveness of colonoscopic surveillance has not been evaluated in an experimental setting. The Manchester approach is more able to detect path_MSH6 cases (and likely more able to detect path_PMS2 cases) than MSI-based testing, but these women are also at lower risk of colorectal cancer, and therefore if colonoscopic surveillance is less effective than generally thought, testing for these cases and providing surveillance may not be cost-effective. 
Our model to extrapolate long-term costs and benefits focusses on colorectal consequences of Lynch syndrome, and does not model future gynaecological cancer or gynaecological surveillance in relatives, nor does it model the cost or effect of aspirin (which may be used to reduce the incidence of Lynch syndrome associated cancers [37]), nor does it model extra-colonic non-gynaecological cancers.

Although the methods employed in this study are broadly applicable, it is important to note that there are frequently challenges to generalising the results of cost-effectiveness analyses to other settings [38].

Author Contributions: Conceptualization, T.M.S., N.A.J.R., and E.J.C.; methodology, T.M.S.; software, T.M.S.; validation, N.A.J.R. and E.J.C.; formal analysis, T.M.S.; investigation, T.M.S.; resources, N.A.J.R. and E.J.C.; data curation, T.M.S. and N.A.J.R.; writing-original draft preparation, T.M.S.; writing-review and editing, N.A.J.R. and E.J.C.; visualization, T.M.S.; supervision, T.M.S.; project administration, T.M.S.; funding acquisition, not applicable. All authors have read and agreed to the published version of the manuscript.

Funding: This research received no specific external funding. N.A.J.R. was supported by a Doctoral Medical Research Council (MRC) Research Fellowship (MR/M018431/1). E.J.C. was supported through the National Institute for Health Research (NIHR) Manchester Biomedical Research Centre (IS-BRC-1215-20007).

Conflicts of Interest: The authors declare no conflict of interest.

\section{Appendix A}

Table A1. Details of model parameters.

\begin{tabular}{|c|c|c|}
\hline Parameter & Base Case Value & Uncertainty (Distribution, $95 \% \mathrm{CI})^{1}$ \\
\hline \multicolumn{3}{|c|}{ Population Characteristics } \\
\hline Prevalence of Lynch syndrome & $3.20 \%$ & Bootstrap, $1.96 \%$ to $5.68 \%$ \\
\hline Prevalence of path_MLH1 & $0.40 \%$ & Bootstrap, $0.03 \%$ to $1.41 \%$ \\
\hline Prevalence of path_MSH2 & $0.80 \%$ & Bootstrap, $0.24 \%$ to $2.03 \%$ \\
\hline Prevalence of path_MSH6 & $1.60 \%$ & Bootstrap, $0.68 \%$ to $3.19 \%$ \\
\hline Prevalence of path_PMS2 & $0.40 \%$ & Bootstrap, $0.02 \%$ to $1.33 \%$ \\
\hline Age of Lynch syndrome cases (years) & 54 & Bootstrap, 47.0 to 60.9 \\
\hline Age of path_MLH1 cases & 40.5 & Bootstrap, 31.4 to 50.2 \\
\hline Age of path_MSH2 cases & 53.8 & Bootstrap, 44.7 to 62.3 \\
\hline Age of path_MSH6 cases & 59.1 & Bootstrap, 49.5 to 67.8 \\
\hline Age of path_PMS2 cases & 47.5 & Bootstrap, 16.6 to 79.5 \\
\hline Age of sporadic cases & 63.5 & Bootstrap, 62.4 to 64.6 \\
\hline Relatives per proband & 6 & Gamma, 1.80 to 11.96 \\
\hline Probability relative accepts counselling & $77.70 \%$ & Beta mixture, $73.8 \%$ to $81.1 \%$ \\
\hline Probability relative accepts testing after counselling & $71.60 \%$ & Ratio, $67.4 \%$ to $76.4 \%$ \\
\hline Probability relative has Lynch syndrome & $44.00 \%$ & Beta, $41.0 \%$ to $47.3 \%$ \\
\hline Probability relative is female & $52.80 \%$ & Beta, $47.6 \%$ to $57.0 \%$ \\
\hline \multicolumn{3}{|c|}{ Diagnostic Effectiveness } \\
\hline Strategy 1 sensitivity & 0.563 & Bootstrap, 0.256 to 0.818 \\
\hline Strategy 1 specificity & 0.835 & Bootstrap, 0.804 to 0.868 \\
\hline Strategy 2 sensitivity & 0.563 & Bootstrap, 0.256 to 0.818 \\
\hline Strategy 2 specificity & 0.967 & Bootstrap, 0.948 to 0.981 \\
\hline Strategy 3 sensitivity & 1 & Bootstrap, 1.000 to 1.000 \\
\hline Strategy 3 specificity & 0.967 & Bootstrap, 0.954 to 0.986 \\
\hline \multicolumn{3}{|c|}{ Disease Natural History } \\
\hline \multicolumn{3}{|c|}{ Colorectal cancer risk for proband aged 60 years to age 80 years 2,3} \\
\hline path_MLH1 & $39.50 \%$ & Log-normal model, $33.5 \%$ to $47.4 \%$ \\
\hline path_MSH2 & $35.70 \%$ & Log-normal model, $28.9 \%$ to $42.1 \%$ \\
\hline path_MSH6 & $19.90 \%$ & Log-normal model, $12.1 \%$ to $28.1 \%$ \\
\hline path_PMS2 & $10.20 \%$ & Log-normal model, $1.3 \%$ to $30.2 \%$ \\
\hline Sporadic & $2.19 \%$ & Not varied \\
\hline \multicolumn{3}{|c|}{ Colorectal cancer risk for female relative aged 60 years to age 80 years 2,3} \\
\hline path_MLH1 & $30.80 \%$ & Log-normal model, $24.1 \%$ to $40.1 \%$ \\
\hline path_MSH2 & $27.00 \%$ & Log-normal model, $20.5 \%$ to $36.3 \%$ \\
\hline path_MSH6 & $12.80 \%$ & Log-normal model, $6.9 \%$ to $22.7 \%$ \\
\hline path_PMS2 & $5.50 \%$ & Log-normal model, $0.5 \%$ to $24.9 \%$ \\
\hline Sporadic & $2.19 \%$ & Not varied \\
\hline
\end{tabular}


Table A2. Details of model parameters.

\begin{tabular}{|c|c|c|}
\hline Parameter & Base Case Value & Uncertainty (Distribution, $95 \% \mathrm{CI})^{1}$ \\
\hline \multicolumn{3}{|c|}{ Colorectal cancer risk for male relative aged 60 years to age 80 years 2,3} \\
\hline path_MLH1 & $35.20 \%$ & Log-normal model, $28.7 \%$ to $43.8 \%$ \\
\hline path_MSH2 & $31.40 \%$ & Log-normal model, $23.8 \%$ to $40.7 \%$ \\
\hline path_MSH6 & $16.30 \%$ & Log-normal model, $9.5 \%$ to $26.2 \%$ \\
\hline path_PMS2 & $7.70 \%$ & Log-normal model, $0.9 \%$ to $30.7 \%$ \\
\hline Sporadic & $3.48 \%$ & Not varied \\
\hline \multicolumn{3}{|c|}{ 5-year endometrial cancer mortality risk for probands ${ }^{3}$} \\
\hline Lynch syndrome cases & $10.40 \%$ & Exponential model, $6.1 \%$ to $15.2 \%$ \\
\hline \multicolumn{3}{|l|}{ Sporadic cases ( $\mathrm{x}=$ age at diagnosis $)$} \\
\hline$x<45$ & $12.40 \%$ & Exponential model, $10.5 \%$ to $14.2 \%$ \\
\hline $45 \leq x<55$ & $13.10 \%$ & Exponential model, $11.9 \%$ to $14.5 \%$ \\
\hline $55 \leq x<65$ & $14.50 \%$ & Exponential model, $13.6 \%$ to $15.4 \%$ \\
\hline $65 \leq x<75$ & $21.50 \%$ & Exponential model, $20.7 \%$ to $22.5 \%$ \\
\hline$x \geq 75$ & $36.90 \%$ & Exponential model, $35.3 \%$ to $38.9 \%$ \\
\hline \multicolumn{3}{|c|}{ 10-year other-cause mortality risk ${ }^{3}$} \\
\hline Woman aged 60 & $7.60 \%$ & Not varied \\
\hline Woman aged 70 & $20.30 \%$ & Not varied \\
\hline Man aged 60 & $11.40 \%$ & Not varied \\
\hline Man aged 70 & $28.10 \%$ & Not varied \\
\hline \multicolumn{3}{|c|}{ 5-year colorectal cancer mortality ${ }^{3}$} \\
\hline \multicolumn{3}{|l|}{ Lynch syndrome case, by stage } \\
\hline Stage I & $4.50 \%$ & Exponential model, $2.1 \%$ to $8.0 \%$ \\
\hline Stage II & $15.80 \%$ & Exponential model, $7.7 \%$ to $27.0 \%$ \\
\hline Stage III & $38.60 \%$ & Exponential model, $20.8 \%$ to $59.2 \%$ \\
\hline Stage IV & $93.40 \%$ & Exponential model, $92.9 \%$ to $93.9 \%$ \\
\hline \multicolumn{3}{|l|}{ Sporadic case, by stage } \\
\hline Stage I & $6.80 \%$ & Exponential model, $6.1 \%$ to $7.5 \%$ \\
\hline Stage II & $22.90 \%$ & Exponential model, $22.5 \%$ to $23.6 \%$ \\
\hline Stage III & $52.30 \%$ & Exponential model, $51.7 \%$ to $53.0 \%$ \\
\hline Stage IV & $93.40 \%$ & Exponential model, $92.9 \%$ to $93.9 \%$ \\
\hline \multicolumn{3}{|c|}{ Colonoscopic Surveillance Effectiveness } \\
\hline $\begin{array}{l}\text { Hazard ratio for incidence of colorectal cancer from } \\
\text { colonoscopic surveillance }\end{array}$ & 0.387 & Log-normal model, 0.164 to 0.753 \\
\hline \multicolumn{3}{|c|}{ Colorectal cancer stage distribution } \\
\hline \multicolumn{3}{|l|}{ Lynch syndrome case, under surveillance } \\
\hline Stage I & $68.60 \%$ & Dirichlet distribution, $52.9 \%$ to $81.8 \%$ \\
\hline Stage II & $10.50 \%$ & Dirichlet distribution, $3.3 \%$ to $22.3 \%$ \\
\hline Stage III & $12.80 \%$ & Dirichlet distribution, $5.0 \%$ to $23.4 \%$ \\
\hline Stage IV & $8.10 \%$ & Dirichlet distribution, $1.8 \%$ to $18.8 \%$ \\
\hline \multicolumn{3}{|l|}{ Lynch syndrome case, not under surveillance } \\
\hline Stage I & $18.80 \%$ & Dirichlet distribution, $9.2 \%$ to $34.2 \%$ \\
\hline Stage II & $48.80 \%$ & Dirichlet distribution, $31.7 \%$ to $63.9 \%$ \\
\hline Stage III & $21.20 \%$ & Dirichlet distribution, $10.7 \%$ to $32.8 \%$ \\
\hline Stage IV & $11.20 \%$ & Dirichlet distribution, $4.1 \%$ to $22.8 \%$ \\
\hline \multicolumn{3}{|l|}{ Sporadic case } \\
\hline Stage I & $17.60 \%$ & Dirichlet distribution, $17.2 \%$ to $18.0 \%$ \\
\hline Stage II & $27.00 \%$ & Dirichlet distribution, $26.5 \%$ to $27.5 \%$ \\
\hline Stage III & $29.50 \%$ & Dirichlet distribution, $29.1 \%$ to $30.0 \%$ \\
\hline Stage IV & $25.90 \%$ & Dirichlet distribution, $25.4 \%$ to $26.4 \%$ \\
\hline \multicolumn{3}{|c|}{ Utility Values } \\
\hline \multicolumn{3}{|c|}{ Baseline utility by gender and age (years) } \\
\hline Woman, 40 & 0.887 & Regression model, 0.826 to 0.949 \\
\hline Woman, 50 & 0.855 & Regression model, 0.766 to 0.944 \\
\hline Woman, 60 & 0.816 & Regression model, 0.696 to 0.937 \\
\hline Woman, 70 & 0.77 & Regression model, 0.611 to 0.931 \\
\hline Woman, 80 & 0.718 & Regression model, 0.513 to 0.924 \\
\hline Woman, 90 & 0.659 & Regression model, 0.403 to 0.917 \\
\hline Man, 40 & 0.909 & Regression model, 0.845 to 0.974 \\
\hline Man, 50 & 0.876 & Regression model, 0.787 to 0.968 \\
\hline Man, 60 & 0.837 & Regression model, 0.717 to 0.963 \\
\hline Man, 70 & 0.791 & Regression model, 0.630 to 0.954 \\
\hline Man, 80 & 0.739 & Regression model, 0.534 to 0.945 \\
\hline Man, 90 & 0.68 & Regression model, 0.424 to 0.938 \\
\hline \multicolumn{3}{|c|}{ Utility modifiers (multipliers) } \\
\hline Stage IV colorectal cancer & 0.789 & Beta, 0.721 to 0.844 \\
\hline
\end{tabular}


Table A3. Details of model parameters.

\begin{tabular}{|c|c|c|}
\hline Parameter & Base Case Value & Uncertainty (Distribution, $95 \% \mathrm{CI})^{1}$ \\
\hline \multicolumn{3}{|c|}{ Costs } \\
\hline \multicolumn{3}{|c|}{ Diagnostic costs } \\
\hline IHC & $£ 30.36$ & Gamma, $£ 21.10$ to $£ 44.95$ \\
\hline MSI & $£ 36.63$ & Gamma, $£ 23.90$ to $£ 55.35$ \\
\hline MLH1 methylation post-IHC & $£ 32.65$ & Gamma, $£ 20.96$ to $£ 44.72$ \\
\hline MLH1 methylation post-MSI & $£ 22.84$ & Gamma, $£ 14.53$ to $£ 35.86$ \\
\hline NGS & $£ 236.35$ & Gamma, $£ 152.85$ to $£ 326.52$ \\
\hline Post-test counselling (proband) & $£ 133.15$ & Gamma, $£ 83.00$ to $£ 185.05$ \\
\hline Consent to test (proband) & $£ 13.64$ & Gamma, $£ 12.60$ to $£ 14.79$ \\
\hline GP referral (relative) & $£ 36.40$ & Gamma, $£ 24.16$ to $£ 48.46$ \\
\hline Pre-test counselling (relative) & $£ 171.73$ & Gamma, $£ 110.07$ to $£ 252.03$ \\
\hline Predictive mutation testing (relative) & $£ 166.32$ & Gamma, $£ 116.27$ to $£ 245.42$ \\
\hline Post-test counselling (relative) & $£ 133.15$ & Gamma, $£ 84.75$ to $£ 183.80$ \\
\hline PREMM $_{5}$ scoring & $£ 3.58$ & Gamma, $£ 2.35$ to $£ 5.29$ \\
\hline \multicolumn{3}{|c|}{ Surveillance costs } \\
\hline Colonoscopy & $£ 583.34$ & Gamma, $£ 383.84$ to $£ 809.88$ \\
\hline Interval between colonoscopies & 2.1 years & Log-normal, 1.54 to 2.89 years \\
\hline \multicolumn{3}{|c|}{ Colorectal cancer costs (lifetime discounted costs, by stage and age) } \\
\hline Stage I, $<50$ & $£ 8754$ & Gamma, $£ 5907$ to $£ 11,966$ \\
\hline Stage I, 50-59 & $£ 5712$ & Gamma, $£ 3793$ to $£ 7993$ \\
\hline Stage I, 60-69 & $£ 4623$ & Gamma, $£ 2990$ to $£ 6602$ \\
\hline Stage I, 70-79 & $£ 3178$ & Gamma, $£ 2125$ to $£ 4701$ \\
\hline Stage I, $\geq 80$ & $£ 1380$ & Gamma, $£ 917$ to $£ 1868$ \\
\hline Stage II, $<50$ & $£ 8741$ & Gamma, $£ 5529$ to $£ 12,172$ \\
\hline Stage II, 50-59 & $£ 7016$ & Gamma, $£ 4367$ to $£ 10,348$ \\
\hline Stage II, 60-69 & $£ 5352$ & Gamma, $£ 3447$ to $£ 7751$ \\
\hline Stage II, 70-79 & $£ 3455$ & Gamma, $£ 2166$ to $£ 5124$ \\
\hline Stage II, $\geq 80$ & $£ 1546$ & Gamma, $£ 923$ to $£ 2216$ \\
\hline Stage III, $<50$ & $£ 14,490$ & Gamma, $£ 8742$ to $£ 21,370$ \\
\hline Stage III, 50-59 & $£ 9692$ & Gamma, $£ 6269$ to $£ 13,227$ \\
\hline Stage III, 60-69 & $£ 7259$ & Gamma, $£ 4500$ to $£ 10,369$ \\
\hline Stage III, 70-79 & $£ 4485$ & Gamma, $£ 2965$ to $£ 6123$ \\
\hline Stage III, $\geq 80$ & $£ 1561$ & Gamma, $£ 1044$ to $£ 2424$ \\
\hline Stage IV,$<50$ & $£ 11,705$ & Gamma, $£ 7719$ to $£ 17,169$ \\
\hline Stage IV, 50-59 & $£ 8444$ & Gamma, $£ 5762$ to $£ 11,688$ \\
\hline Stage IV, 60-69 & $£ 6509$ & Gamma, $£ 4461$ to $£ 9038$ \\
\hline Stage IV, 70-79 & $£ 4365$ & Gamma, $£ 2636$ to $£ 5946$ \\
\hline Stage IV,$\geq 80$ & $£ 807$ & Gamma, $£ 514$ to $£ 1097$ \\
\hline
\end{tabular}

${ }^{1}$ Bootstrap 95\% confidence intervals calculated using the normal approximation on a suitable transformed scale (logit was preferred for proportions but arcsine was used when bootstrap iterations included 0 or 1 ). ${ }^{2}$ Estimates for those with Lynch syndrome are in the presence of colonoscopic surveillance. ${ }^{3}$ Calculations conducted absent competing risks.

\section{References}

1. Vasen, H.; Blanco, I.; Aktan-Collan, K.; Gopie, J.P.; Alonso, A.; Aretz, S.; Bernstein, I.T.; Bertario, L.; Burn, J.; Capellá, G.; et al. Revised guidelines for the clinical management of Lynch syndrome (HNPCC): Recommendations by a group of European experts. Gut 2013, 62, 812-823. [CrossRef] [PubMed]

2. Hampel, H.; Frankel, W.; Panescu, J.; Lockman, J.; Sotamaa, K.; Fix, D.; Comeras, I.; La Jeunesse, J.; Nakagawa, H.; Westman, J.A.; et al. Screening for Lynch Syndrome (Hereditary Nonpolyposis Colorectal Cancer) among Endometrial Cancer Patients. Cancer Res. 2006, 66, 7810-7817. [CrossRef] [PubMed]

3. Hampel, H.; Frankel, W.L.; Martin, E.; Arnold, M.; Khanduja, K.; Kuebler, P.; Clendenning, M.; Sotamaa, K.; Prior, T.; Westman, J.A.; et al. Feasibility of Screening for Lynch Syndrome Among Patients With Colorectal Cancer. J. Clin. Oncol. 2008, 26, 5783-5788. [CrossRef] [PubMed]

4. Grosse, S.D. When is Genomic Testing Cost-Effective? Testing for Lynch Syndrome in Patients with Newly-Diagnosed Colorectal Cancer and Their Relatives. Healthcare 2015, 3, 860-878. [CrossRef] [PubMed]

5. Ladabaum, U.; Wang, G.; Terdiman, J.; Blanco, A.; Kuppermann, M.; Boland, C.R.; Ford, J.; Elkin, E.; Phillips, K.A. Strategies to Identify the Lynch Syndrome Among Patients with Colorectal Cancer. Ann. Intern. Med. 2011, 155, 69-79. [CrossRef] [PubMed]

6. Mvundura, M.; Grosse, S.D.; Hampel, H.; Palomaki, G.E. The cost-effectiveness of genetic testing strategies for Lynch syndrome among newly diagnosed patients with colorectal cancer. Genet. Med. 2009, 12, 93-104. [CrossRef] 
7. Snowsill, T.; Coelho, H.; Huxley, N.; Jones-Hughes, T.; Briscoe, S.; Frayling, I.M.; Hyde, C. Molecular testing for Lynch syndrome in people with colorectal cancer: Systematic reviews and economic evaluation. Health Technol. Assess. 2017, 21, 1-238. [CrossRef]

8. Snowsill, T.; Huxley, N.; Hoyle, M.; Jones-Hughes, T.; Coelho, H.; Cooper, C.; Frayling, I.M.; Hyde, C. A systematic review and economic evaluation of diagnostic strategies for Lynch syndrome. Health Technol. Assess. 2014, 18, 1-406. [CrossRef]

9. Bruegl, A.S.; Djordjevic, B.; Batte, B.; Daniels, M.; Fellman, B.; Urbauer, D.; Luthra, R.; Sun, C.; Lu, K.H.; Broaddus, R.R. Evaluation of clinical criteria for the identification of Lynch syndrome among unselected patients with endometrial cancer. Cancer Prev. Res. 2014, 7, 686-697. [CrossRef]

10. Goverde, A.; Spaander, M.C.; Van Doorn, H.C.; Dubbink, H.J.; Ouweland, A.M.V.D.; Tops, C.M.; Kooi, S.G.; De Waard, J.; Hoedemaeker, R.F.; Bruno, M.; et al. Cost-effectiveness of routine screening for Lynch syndrome in endometrial cancer patients up to 70 years of age. Gynecol. Oncol. 2016, 143, 453-459. [CrossRef]

11. Kwon, J.S.; Scott, J.L.; Gilks, C.B.; Daniels, M.; Sun, C.C.; Lu, K.H. Testing Women with Endometrial Cancer to Detect Lynch Syndrome. J. Clin. Oncol. 2011, 29, 2247-2252. [CrossRef] [PubMed]

12. Resnick, K.; Straughn, J.M., Jr.; Backes, F.; Hampel, H.; Matthews, K.S.; Cohn, D.E. Lynch syndrome screening strategies among newly diagnosed endometrial cancer patients. Obstet. Gynecol. 2009, 114, 530-536. [CrossRef] [PubMed]

13. Snowsill, T.M.; Ryan, N.A.J.; Crosbie, E.J.; Frayling, I.M.; Evans, D.G.; Hyde, C.J. Cost-effectiveness analysis of reflex testing for Lynch syndrome in women with endometrial cancer in the UK setting. PLoS ONE 2019, 14, e221419. [CrossRef] [PubMed]

14. Nakagawa, H.; Nuovo, G.J.; Zervos, E.E.; Martin, E.W.; Salovaara, R.; Aaltonen, L.A.; de la Chapelle, A. Age-related Hypermethylation of the $5^{\prime}$ Region of MLH1 in Normal Colonic Mucosa Is Associated with Microsatellite-unstable Colorectal Cancer Development. Cancer Res. 2001, 61, 6991.

15. Wu, Y.; Berends, M.J.W.; Mensink, R.G.J.; Kempinga, C.; Sijmons, R.; Van Der Zee, A.G.J.; Hollema, H.; Kleibeuker, J.H.; Buys, C.H.C.M.; Hofstra, R. Association of Hereditary Nonpolyposis Colorectal Cancer-Related Tumors Displaying Low Microsatellite Instability with MSH6 Germline Mutations. Am. J. Hum. Genet. 1999, 65, 1291-1298. [CrossRef]

16. Ryan, N.; McMahon, R.; Tobi, S.; Snowsill, T.; Esquibel, S.; Wallace, A.; Bunstone, S.; Bowers, N.; Mosneag, I.; Kitson, S.; et al. The Proportion of Endometrial Tumors Associated with Lynch Syndrome (PETALS study). PLOS Med. (Under review).

17. Kastrinos, F.; Uno, H.; Ukaegbu, C.; Alvero, C.; McFarland, A.; Yurgelun, M.B.; Kulke, M.H.; Schrag, D.; Meyerhardt, J.A.; Fuchs, C.S.; et al. Development and Validation of the PREMM5 Model for Comprehensive Risk Assessment of Lynch Syndrome. J. Clin. Oncol. 2017, 35, 2165-2172. [CrossRef]

18. Anonymous. The R Project for Statistical Computing. Available online: http://www.r-project.org/ (accessed on 13 February 2012).

19. Filipovic-Pierucci, A.; Zarca, K.; Durand-Zaleski, I. Markov Models for Health Economic Evaluations: The R Package heemod. Value Health 2017, 19, 369. [CrossRef]

20. Järvinen, H.J.; Aarnio, M.; Mustonen, H.; Aktan-Collan, K.; Aaltonen, L.; Peltomaki, P.; De La Chapelle§, A.; Mecklin, J. Controlled 15-year trial on screening for colorectal cancer in families with hereditary nonpolyposis colorectal cancer. Gastroenterology 2000, 118, 829-834. [CrossRef]

21. Mecklin, J.-P.; Aarnio, M.; Läärä, E.; Kairaluoma, M.V.; Pylvänäinen, K.; Peltomaki, P.; Aaltonen, L.; Järvinen, H.J. Development of Colorectal Tumors in Colonoscopic Surveillance in Lynch Syndrome. Gastroenterology 2007, 133, 1093-1098. [CrossRef]

22. Curtis, L.; Burns, A. Unit Costs of Health and Social Care 2018; Personal Social Services Research Unit (PSSRU); University of Kent: Caterbury, Kent, UK, 2018. [CrossRef]

23. Ryan, N.A.J.; Davison, N.; Payne, K.; Cole, A.; Evans, D.G.; Crosbie, E.J. A Micro-Costing Study of Screening for Lynch Syndrome-Associated Pathogenic Variants in an Unselected Endometrial Cancer Population: Cheap as NGS Chips? Front. Oncol. 2019, 9, 61. [CrossRef] [PubMed]

24. Slade, I.; Hanson, H.; George, A.; Kohut, K.; Strydom, A.; Wordsworth, S.; Rahman, N.; Programme, M. A cost analysis of a cancer genetic service model in the UK. J. Community Genet. 2016, 7, 185-194. [CrossRef]

25. Rahman, N. Mainstreaming genetic testing of cancer predisposition genes. Clin. Med. 2014, 14, 436-439. [CrossRef] [PubMed] 
26. NHS Improvement. National Schedule of Reference Costs 2016-2017. Available online: https://improvement. nhs.uk/resources/reference-costs/ (accessed on 3 April 2020).

27. Department of Health. NHS Reference Costs 2015 to 2016. Available online: https://www.gov.uk/government/ publications/nhs-reference-costs-2015-to-2016 (accessed on 6 December 2017).

28. Whyte, S.; Harnan, S.; Scope, A.; Simpson, E.; Tappenden, P.; Duffy, S.; Rachet, B.; Sculpher, M.; Hinde, S.; McKenna, C.; et al. Early Awareness Interventions for Cancer: Colorectal Cancer; Economic Evaluation of Health and Social Care Interventions Policy Research Unit: University of Sheffield; University of York: Sheffield, UK; York, UK, 2012.

29. Ara, R.; Brazier, J. Populating an Economic Model with Health State Utility Values: Moving toward Better Practice. Value Health 2010, 13, 509-518. [CrossRef] [PubMed]

30. Djalalov, S.; Rabeneck, L.; Tomlinson, G.; Bremner, K.E.; Hilsden, R.; Hoch, J.S. A Review and Meta-analysis of Colorectal Cancer Utilities. Med. Decis. Mak. 2014, 34, 809-818. [CrossRef]

31. Arrigoni, A.; Sprujevnik, T.; Alvisi, V.; Rossi, A.; Ricci, G.; Pennazio, M.; Spandre, M.; Cavallero, M.; Bertone, A.; Foco, A.; et al. Clinical identification and long-term surveillance of 22 hereditary non-polyposis colon cancer Italian families. Eur. J. Gastroenterol. Hepatol. 2005, 17, 213-219. [CrossRef]

32. Ladabaum, U.; Ford, J.M.; Martel, M.; Barkun, A.N. American Gastroenterological Association Technical Review on the Diagnosis and Management of Lynch Syndrome. Gastroenterology 2015, 149, 783-813. [CrossRef]

33. Møller, P.; Seppälä, T.; Bernstein, I.T.; Holinski-Feder, E.; Sala, P.; Evans, D.G.; Lindblom, A.; Macrae, F.; Blanco, I.; Sijmons, R.H.; et al. Cancer risk and survival in path_MMR carriers by gene and gender up to 75 years of age: A report from the Prospective Lynch Syndrome Database. Gut 2017, 67, 1306-1316. [CrossRef]

34. Ryan, N.A.J.; Morris, J.; Green, K.; Lalloo, F.; Woodward, E.R.; Hill, J.; Crosbie, E.J.; Evans, D.G. Association of Mismatch Repair Mutation with Age at Cancer Onset in Lynch Syndrome. JAMA Oncol. 2017, 3, 1702-1706. [CrossRef]

35. Crim, A.; Perkins, V.; Husain, S.; Ding, K.; Holman, L. Feasibility of two-antibody vs four-antibody mismatch repair protein immunohistochemistry as initial screening for Lynch syndrome in patients with endometrial adenocarcinoma. Gynecol. Oncol. 2017, 145, 44. [CrossRef]

36. Kausmeyer, D.T.; Lengerich, E.J.; Kluhsman, B.C.; Morrone, D.; Harper, G.R.; Baker, M.J. A Survey of Patients' Experiences with the Cancer Genetic Counseling Process: Recommendations for Cancer Genetics Programs. J. Genet. Couns. 2006, 15, 409-431. [CrossRef] [PubMed]

37. Burn, J.; Gerdes, A.-M.; Macrae, F.; Mecklin, J.-P.; Moeslein, G.; Olschwang, S.; Eccles, D.M.; Evans, D.G.R.; Maher, E.R.; Bertario, L.; et al. Long-term effect of aspirin on cancer risk in carriers of hereditary colorectal cancer: An analysis from the CAPP2 randomised controlled trial. Lancet 2011, 378, 2081-2087. [CrossRef]

38. Sculpher, M.J.; Pang, F.S.; Manca, A.; Drummond, M.F.; Golder, S.; Urdahl, H.; Davies, L.; Eastwood, A. Generalisability in economic evaluation studies in healthcare: A review and case studies. Health Technol. Assess. 2004, 8, 8. [CrossRef] [PubMed]

(C) 2020 by the authors. Licensee MDPI, Basel, Switzerland. This article is an open access article distributed under the terms and conditions of the Creative Commons Attribution (CC BY) license (http://creativecommons.org/licenses/by/4.0/). 